\title{
A Dynamic Model of the Tragedy of the Commons in Marketing-Intensive Industries
}

\author{
Dohoon Kim \\ School of Management, Kyung Hee University, 26, Kyungheedae-ro, Dongdaemun-gu, Seoul 130-701, Republic of Korea \\ Correspondence should be addressed to Dohoon Kim; dyohaan@khu.ac.kr
}

Received 18 January 2014; Revised 17 March 2014; Accepted 18 March 2014; Published 27 April 2014

Academic Editor: Nachamada Blamah

Copyright ( 2014 Dohoon Kim. This is an open access article distributed under the Creative Commons Attribution License, which permits unrestricted use, distribution, and reproduction in any medium, provided the original work is properly cited.

\begin{abstract}
This study provides a dynamic model and analyzes its process that may plunge the business ecosystem into ToC (the Tragedy of the Commons). When developing the model, we have in mind some industries where the marketing competition to secure a large installed base is intense. The social commerce industry is a representative example of this type of industries, but the scope of this study is not limited to the industry. We first introduce a previous study focusing on the static Nash equilibrium, and then present an extended version of the basic model in a dynamic perspective. According to our analyses on the dynamic equilibria together with their stability, there may be a unique interior equilibrium, but it is highly likely unstable. In addition, possible (near) boundary equilibria are also unstable for a wide range of parameter values. We also conduct some numerical experiments and discover cycles as solutions to some particular instances. Since those cycles contain the ToC traps, a policy measure or regulation may need to be employed. Our approach and results will help to figure out a clue to escape from the ToC trap, thereby shedding new light on the sustainable growth of the business ecosystem, which is prone to excessive marketing competition.
\end{abstract}

\section{Introduction}

As the mobile services and SNS (social network service) are becoming the most common and popular media to access and use the Internet, they are rapidly replacing PCs and other types of information devices dedicated to serve a specific purpose. Information flows through social media keep increasing and will change the way of organizing and leading businesses and industries. For example, marketing practice has entered a new horizon, where mobile social media plays a central role in firms' marketing campaign and reduces the entry barrier by expanding the spheres of activities and lowering the access costs. One can find representative cases in the social commerce industry which utilizes social media as a commercial platform. It is not surprising to discover a strong incentive to construct a business model with Facebook and Twitter, each of which retains more than 1 billion and 5,000 million users over the globe, respectively.

Despite their tremendous success in creating new service markets and expanding the business areas, SNS platforms and other e-commerce providers based on social media face many challenges in practice. In particular, the social commerce providers have experienced rise and fall over a short period of time right after their beginning (MacMillan [1-3], Reibstein [4], Webster [5], and Wheeler [6]). Indeed, the number of providers is decreasing in many countries. Groupon, the first global social commerce company, went through hardship in its IPO (initial public offering) and suffered sharp drops of its stock price right after the IPO.

These problems and challenges are not found only in the social commerce area. In effect, when innovators and pioneers plan to monetize eyeballs, they are likely to be exposed to the risk that arises from those problems. In that sense, the issue may be generally embedded or innate property of many business areas, which directly or indirectly depend on the installed base (Kumar and Rajan [7] and Patel [8]). Even for Facebook and Twitter, for example, the dream of advertising revenue has yet to be substantially realized, and many potential investors are still stuck at the thinking that if these providers could secure a large number of users, they would be able to sell new products and services to their users in the future (Cusumano [9], Kruschwitz [10], and Leber [11]). 
If this is the case, the entire industry is highly likely to plunge into a marketing competition, where each player competes to expand its prospective installed base by reinforcing marketing activities in a broad sense. SNS, social commerce, online/mobile games, telecom services, and many ICT business areas experience and suffer from marketing wars fighting for customers and users.

One of the major backgrounds of the marketing competition comes from the cost structure of the corresponding industry. For example, a low entry barrier as in the case of social commerce (Anderson et al. [12], Reibstein [4], Wheeler [6], and Urstadt [13]) makes it possible for a great number of potential players to enter the industry once they observe a positive gain above the normal profits. Seeking the first mover's advantage may be another driver of the marketing war. In such a case, relatively few competing providers spend lots of cash on advertising activities. Telecom operators and pharmaceutical companies are the best example of this case. We will call this type of industries the "marketing-intensive industry."

However, it is not easy to find analytical studies about the marketing competition of this kind. One major reason for the meager literature on this inherent weakness of the marketing-intensive industries can be found in a tradition of economic studies. That is, firms and providers are typically assumed to seek for the profit, not for the market share. But, recent changes occurring across the broad range of industries defy this tradition. One of them is fusion or convergence across multiple industries, which conceals the exact financial gain of a company participating in a business ecosystem built upon multiple industries. Another big change is referred to as "servicification," which emphasizes the trend that services are increasingly essential as both inputs and outputs in many industries including even traditional manufacturing areas such as automobile and consumer electronics (Lodefalk [14], Schmitt and Hatfield [15], and Stephen and Toubia [16]). The ICT industries, particularly social media services, experience both trends, thereby exposing themselves to fierce competition for market share.

This study suggests a modeling framework for and conducts analysis of the marketing-intensive industries vulnerable to the risk of collapse due to excessive competition to expand the installed base. Such industries are prone to getting mired in marketing wars due to inherent properties in the business model itself (Though we started this section with mentioning the social media industry as one of representative instances that fit our model assumptions, the application scope of our approach will not be confined to social media and other ICT industries). We first develop a stylized business model that captures the essential features of the competition process. Our approach focuses on the relationship between key decision issues such as marketing inputs and market value. As more providers join the industry thanks to the low entry barrier, they are inevitably faced with fierce competition. This may lead to sharp increase in the expenditure related to marketing and advertising activities in a broad sense. This type of competition may lead the industry away from its optimal development path and, at worst, toward a collapse of the entire business ecosystem as described in some ICT business cases above.

Having such a case, the situation that we will deal with in this study resembles $\mathrm{ToC}$ (the tragedy of the commons; Alroy [17], Hardin [18], Greco and Floridi [19], and Knowledge at Wharton [20]), where the user market (the commons in our metaphor) is vulnerable to exploitation by providers' aggressive marketing to enhance their installed base. Thus, another goal of this study should be to examine the possibilities and conditions that ToC may occur in such a business ecosystem. In fact, oscillations and/or emergence of $\mathrm{ToC}$ are not a special case but rather common in dynamic formulations. They have been observed in many different circumstances, in particular, with a structured population (Perc and Szolnoki [21], Perc et al. [22], Santos et al. [23], Szolnoki and Perc [24], Szolnoki and Perc [25], and Szolnoki and Perc [26]). However, such a dynamic nature not unusual in natural phenomenon may also prevail over stable equilibria, particularly in the business areas characterized by severe marketing competition on the basis of a perfectly mixed population. If it is true, we should examine the possibility of unintended consequences of an excessive competition as it is likely to impinge upon market maturity. For that purpose, we will analyze the process that plunges the business ecosystem into $\mathrm{ToC}$ and conduct some experiments in order to investigate the effects of the parameters in our model. Our approach and expected results will help to discover some clues to escape from this kind of traps, thereby shedding new light on the sustainable growth of the marketing-intensive industries such as the social media businesses. We will also present some policy implications that could be attained in the course of implementation of our suggestions to alleviate the risk of ToC.

In one of the previous researches on this subject, Kim [27] presents static game models that deal with homogeneous as well as heterogeneous providers in the social commerce industry. In this study, we will also build game models with heterogeneous providers and derive explicit equilibria in a dynamic setting. This study primarily takes the social media industries into account but is not confined within a specific industry as in Kim's study [27]. In particular, the dynamics adopted in our model will show sharp contrasts between static and dynamic approaches in terms of the equilibria, stability, and their resulting insights.

This paper is organized as follows. The next section introduces the basic model and static analysis. Section 3 provides our dynamic model and analysis and compares the results with ones from the static case. In the next section, we present some experiment outcomes together with their lessons and policy implications. Our final section concludes this study and suggests some future works.

\section{Basic Model and Analysis}

2.1. Basic Model. Let us suppose a player set composed of $N$ potential SC providers, where $j$ is employed as the index for an individual (sometimes representative) SC provider. $\mathbf{N}$ denotes the set of SC providers; that is, $\mathbf{N}=\{1, \ldots, N\}$. 
Among $N$ providers, some providers may not join the market. The strategic decision variable of SC provider $j$ is its marketing efforts $e_{j}$. Those who do not join the market exercise 0 marketing efforts.

In our model, SC providers are assumed to be horizontally differentiated according to their marketing capabilities, which is the major factor characterizing the providers. We employ $q_{j}$ to represent the overall marketing costs of provider $j$ and assume that $q_{j}$ 's are inversely proportional to the characteristic of marketing efficiency. Furthermore, the potential providers in $\mathbf{N}$ are given their index in an ascending order based on this attribute. Accordingly, the lower the index is, the more efficient it is in marketing; for example, the first provider in $\mathbf{N}$ (i.e., $j=1$ ) bears the lowest marketing cost $q_{1}$.

In each time period, the entire value of the SC market, $G$, is determined by two factors. First, when all the providers in a subset $P$ of $\mathbf{N}$ (i.e., $P \subseteq \mathbf{N}$ ) are active in the market, the total marketing efforts $E\left(=\sum_{j \in P} e_{j}\right)$ contribute to the market value $G$ by stimulating consumers and boosting the market. However, $E$ also exhibits a negative effect on $G$ since too much or too little marketing efforts over- or underexploit the market. Thus, a deviation from the optimal level of marketing efforts in a certain situation may harm the market conditions in the following period (refer to (1) below).

Second, the entire value of the SC market is also affected by environmental factors such as consumer preference change and technological development. We abbreviate these environmental factors into $M$ as in (1), which will be called "the market maturity factor." However, $M$ will not be assumed to be given exogenously as in Kim's study [27]. Instead, we will develop a dynamic model where $M$ is endogenously determined based on the providers' decisions (refer to Section 3).

As $M$ and $E$ change from one period to another, $G$ also experiences a series of changes. We incorporate the following relationship between the market value and two major factors explained above:

$$
G=G(E \mid M)=M \cdot E \cdot(2 K-E),
$$

where $K$ in (1) represents the optimal level of the total marketing efforts $E^{*}$ embedded (but probably hidden) in the SC industry. Accordingly, (1) implies that a total marketing effort lower than $K$ leaves a room for further expansion of the market, while a total marketing efforts higher than $K$ incurs an overheating and entails a contraction of the market in the following periods.

The market value $G$ realized in the current period is distributed over the active SC providers in the corresponding period. We assume that the allocation of $G$ is proportional to the marketing efforts $e_{j}$ 's. Therefore, the share of the active provider $j, g_{j}$, is determined by $G\left(e_{j} / E\right)=M \cdot(2 K-E) \cdot e_{j}$. Then, the net benefit of the provider $j, r_{j}$, is as follows:

$$
r_{j}=g_{j}-q_{j} \cdot e_{j}=\left\{M(2 K-E)-q_{j}\right\} \cdot e_{j} .
$$

Those who decide not to join the market (i.e., $e_{j}=0$ ) get 0 payoff. Others who participate in the market try to predict the total marketing efforts $E$ and determine their marketing efforts $e_{j}$ 's, each of which maximizes the corresponding $r_{j}$. Unfortunately, however, possible miss predictions result in losses for some providers, that is, resulting $r_{j}<0$ for some providers. In the next period, the providers who experienced losses in the former period will leave the market or try to minimize its loss. In our model, once a provider decides to join the market at $t$, it cannot retreat at $t$. Therefore, when a provider experiences a negative payoff, the provider chooses the marketing level which actually minimizes its loss. Once the number of active providers $P(t)=p$ is determined, one can show that providers' optimal marketing efforts are explicitly determined as in the following section.

2.2. Static Analysis. This section introduces Nash equilibria of the static model based on the previous study of Kim [27]. Before presenting some key results in Kim's study [27], let us define some notions and terms for ease of explanation and enhanced readability:

$$
\begin{aligned}
& \Theta_{n}=\left[\begin{array}{ccccc}
1 & \frac{1}{2} & \frac{1}{2} & \cdots & \frac{1}{2} \\
\frac{1}{2} & 1 & \frac{1}{2} & \cdots & \frac{1}{2} \\
\vdots & & \ddots & & \vdots \\
\frac{1}{2} & \frac{1}{2} & \frac{1}{2} & \cdots & 1
\end{array}\right], \quad \mu_{n}=\left[\begin{array}{c}
K-\frac{q_{1}}{2 M} \\
K-\frac{q_{2}}{2 M} \\
\vdots \\
K-\frac{q_{n}}{2 M}
\end{array}\right], \\
& Q(n)=\sum_{j=1}^{n} q_{j}, \quad E(n)=\sum_{j=1}^{n} e_{j},
\end{aligned}
$$

where $\Theta_{n}$ is $(n \times n)$ square matrix and $\mu_{n}$ is $n$-dimensional column vector whose elements start with $j=1$ in the increasing order. By the property of the symmetric matrix, the inverse matrix of $\Theta_{n}, \Theta_{n}^{-1}$, is also symmetric and derived as follows:

$$
\Theta_{n}^{-1}=\left[\begin{array}{ccccc}
\frac{2 n}{n+1} & \frac{-2}{n+1} & \frac{-2}{n+1} & \cdots & \frac{-2}{n+1} \\
\frac{-2}{n+1} & \frac{2 n}{n+1} & \frac{-2}{n+1} & \cdots & \frac{-2}{n+1} \\
\vdots & & & \ddots & \vdots \\
\frac{-2}{n+1} & \frac{-2}{n+1} & \frac{-2}{n+1} & \cdots & \frac{2 n}{n+1}
\end{array}\right] .
$$

Proposition 1 (see Kim's study [27]). For readability, one omits $t$ in $M(t)$ and $P(t)$. Suppose that there are $P(t)=p$ providers as in the increasing order of $q_{j}$ 's. That is, $p$ providers whose indices spread from 1 to $p$ in $\mathbf{N}$ now enter the market at $t$. Let $y(\leq p)$ denote the index of the provider with $q_{y} \leq$ $(2 K M+Q(p)) /(p+1)$ and $q_{y+1}>(2 K M+Q(p)) /(p+1)$. Then, the marketing effort profile $\left(\bar{e}_{1}, \ldots, \bar{e}_{y}, 0, \ldots, 0\right)$, where $\bar{e}_{j}$ 's are determined as follows, constitute a Nash equilibrium:

$$
\bar{e}_{j}=\left\{\Theta_{y}^{-1} \cdot \mu_{y}\right\}_{j}=2\left(K-\frac{q_{j}}{2 M}\right)-\frac{2 p K}{p+1}+\frac{Q(p)}{M(p+1)},
$$

where $\{\mathbf{x}\}_{j}$ represents the $j$ th element of the vector $\mathbf{x}$.

Proof. Omitted (for proof,see Kim’s study [27]). 
Proposition 1 presents a Nash equilibrium of the basic static model. Note that there is marginal provider represented by $y$. Then, those who have lower index than $y$ earn positive profits (i.e., $r_{j}>0$, for all $j \leq y$ ), while providers whose indices are larger than $y$ do not join the industry, thereby earning nothing (i.e., $r_{j}=0$, for all $j>y$ ). The size of active providers is a function of $M(t)$ and $Q(p)$. Let us call $y$ in Proposition 1 "the efficiency threshold" since $y$ sets the upper bound on the number of active providers, each of which maintains a positive payoff. From Proposition 1 above, we also know that $\bar{E}=\sum_{j=1}^{p} \overline{e_{j}}$ is described by the following equation:

$$
\bar{E}(t)=\frac{2 y K}{y+1}-\frac{Q(y)}{(y+1) M(t)}=\frac{2 y K M(t)-Q(y)}{(y+1) M(t)} .
$$

When all the potential providers are homogeneous (i.e., $q_{j}=$ $q$, for all $j$ ), their strategies will be symmetric and result in a symmetric Nash equilibrium as follows.

Corollary 2 (symmetric equilibrium with homogeneous players). Let one considers the situation where all the providers are homogeneous in the sense of $q_{j}=q$, for all $j \in N$. Suppose that the following inequality holds; that is, $2 M(t) K(t)>q$. Then, at a symmetric equilibrium, all the potential providers join the market and exercise the same marketing effort $\bar{e}$ determined as follows:

$$
\bar{e}_{j}=\bar{e}=\frac{2}{N+1}\left(K-\frac{q}{2 M(t)}\right) \quad \forall j \in \mathbf{N} .
$$

Proof. Omitted (for proof, see Kim's study [27]).

In the case of homogeneous players, we have much simpler expressions for the equilibrium strategies as above. First note that all the homogeneous players join the market or leave the market at the same time. Thus, $y(t)$ in Proposition 1 will be either 0 or $N$, which establishes a key difference from the case of heterogeneous players. With the same $K$ and $M(t)$, the marketing effort of a heterogeneous active player $\left(\bar{e}_{j}\right)$ will be higher than that of a homogeneous player $(\bar{e})$ in general (Of course, the marketing effort of a heterogeneous player close to the marginal provider may be lower than $\bar{e}$ ). The total marketing efforts in the homogeneous case amount to $N \bar{e}$. Subsequently, if $N q<Q(y)$, then the total marketing efforts in the homogeneous case are larger than those in the heterogeneous case. If they are not, however, one cannot uniformly determine the size comparison; it depends on the distribution of $q_{j}$ 's.

\section{Dynamic Model and Analysis}

3.1. Dynamic Model. Now, we will extend the basic model together with its context from a new dynamic perspective. The analytical results above have been derived under the assumption that a certain number of providers ( $p$ providers) already exist in the industry and the market maturity factor $M$ is given and fixed. However, these two parameters ( $p$ and $M$ ) should also change in their turn after the agent behaviors (here, e.g., the best efforts $\bar{e}_{j}$ 's in the Nash equilibrium) are determined. Our dynamic model will reflect these interactions between parameters and endogenous variables in the basic model. In particular, we incorporate the dynamics described below.

First note that it is natural as well as practical to set up the circumstances where the (potential) providers know only the parameter values in the last period. If this is the case, then the active providers try to predict the total marketing efforts $E$ and adjust their marketing efforts $e_{j}$ 's, which optimizes their net benefits and results in nonnegative payoffs. However, their expectations and decisions do not always succeed, and some providers may receive negative payoffs at the end of the corresponding period. Those who experience a negative payoff in the current period will leave the industry in the next period. On the other hand, all the active providers enjoy positive payoffs at $t$ and some potential providers inactive at $t$ will try to join the market at $t+1$.

According to Proposition 1 with given $K$ and $M(t)$ at $t, y$ sets the maximum number of the active providers, each of which earns nonnegative payoff from positive marketing efforts. Though the relationship between $y$ and other parameters is implicitly determined, for a particular $q_{j}$ distribution such as $q_{j}=h \cdot j$ (i.e., marketing effort costs linearly increasing with a positive constant $h$ ), we can explicitly determine $y$ as a function of $M(t)$ and $P(t)$; for example, with the $q_{j}$ specified above, $Q(p)=(p(p+1) / 2) h$ and $y=\lfloor(2 K M / h(p+1))+$ $(p / 2)\rfloor=\lfloor(4 K M+h p(p+1)) / 2 h(p+1)\rfloor$, where $\lfloor x\rfloor$ rounds $x$ down to the nearest integer. Furthermore, by pretending that $y$ is continuous rather than discrete, one can approximate $y(t)$ and, by plugging $Q(p)$ into (6), one can approximate $\bar{E}(t)$ as follows:

$$
\begin{gathered}
y(t) \cong \frac{2 K M(t)}{h P(t)}+\frac{P(t)}{2}, \\
\bar{E}(t) \cong 2 K-\frac{h^{2}}{4 K}\left\{\frac{P(t)}{M(t)}\right\}^{2} .
\end{gathered}
$$

We establish a set of dynamics driven by both the market maturity factor $M(t)$ and the number of active providers $P(t)$. That is, as a result of individual decisions of whether to join the market or not, both $M(t)$ and $P(t)$ are endogenously determined by the following systems of difference equations. We employ a set of adaptive dynamics where $\alpha$ and $\beta$ represent the weights associated with the current states of the active players and the market maturity, respectively. Consider

$$
\begin{aligned}
& P(t+1)=\{\alpha P(t)+(1-\alpha)(y(t)-P(t)) \wedge N\} \vee 0, \\
& M(t+1)=\left\{\beta M(t)+(1-\beta)(K-\bar{E}(t)) \wedge M_{\max }\right\} \vee 0,
\end{aligned}
$$

where $x \wedge y=\min \{x, y\}, x \vee y=\max \{x, y\}$, and $M_{\max }$ represents the maximum level of $M(t)$.

Plugging ((8a) and (8b)) into ((9a) and (9b)) and rearranging the terms, we establish the systems of the following dynamic equations:

$$
\begin{aligned}
P(t+1)= & \left\{\alpha P(t)+(1-\alpha)\left[\frac{2 K M(t)}{h P(t)}-\frac{P(t)}{2}\right]\right. \\
\wedge N\} \vee 0, &
\end{aligned}
$$




$$
\begin{aligned}
M(t+1)= & \left\{\beta M(t)+(1-\beta) K\left[\left(\frac{h P(t)}{2 K M(t)}\right)^{2}-1\right]\right. \\
& \left.\wedge M_{\max }\right\} \vee 0 .
\end{aligned}
$$

Equation (10a) explains the changes in the number of active providers. The size of active providers in the next period $P(t+1)$ will increase if the current $P(t)$ is smaller than $y(t)$, the efficiency threshold (i.e., the maximum number of efficient providers). On the other hand, $P(t+1)$ will decrease if the current number of providers exceeds this threshold since some providers (most likely the ones with low marketing capabilities or high marketing costs) will experience a loss, thereby leaving the industry next time. Lastly, the adaptive dynamics are completed with $\alpha$ as the associated weight. Equation (10b) also follows the similar reasoning as in (10a). It represents the adaptive adjustment process of $M(t)$ (with $\beta$ as its associated weight) when the current total marketing level $\bar{E}(t)$ fails to hit the optimal level of marketing efforts $K$. If the total marketing efforts from the current active providers exceed (in the case of excessive marketing), the market is overexploited and $M(t+1)$ decreases.

According to the system dynamics described in ((10a) and (10b)), a change in $M(t)$ affects the market value $G(t)$ (refer to (1)), which in turn influences the gains of the providers. As a result, some incumbent players exit or some new players enter the industry in the next period, and eventually the total marketing efforts are highly likely to vary. Thus, the possible difference in the speeds of the adjustments may result in a fluctuation in the number of providers, which may hinder the system from settling down to a steady state in the long-run. We will investigate this possibility together with the system performance such as the deviation of the total marketing efforts from the socially optimal level of the marketing.

3.2. Dynamic Analysis. Let us first define a positive constant $\Phi \equiv \sqrt{K^{2}+(4 / 3) h}-K$ to simplify the expressions hereafter. Proposition 3 shows an interior fixed point from the system dynamics. Proposition 4 provides the conditions that the interior fixed point (if exists) is stable or unstable.

Proposition 3. Suppose that both $\alpha$ and $\beta$ belong to $(0,1)$. The following state $(\bar{P}, \bar{M})$ gives an interior fixed point of the system dynamics in ((10a) and (10b)):

$$
\bar{M}=\frac{\Phi}{2}, \quad \bar{P}=\sqrt{\frac{2 K \Phi}{3 h}} .
$$

Proof. The interior equilibrium comes from the solution of the following simultaneous equation system:

$$
\begin{gathered}
P=\alpha P+(1-\alpha)\left[\frac{2 K M}{h P}-\frac{P}{2}\right], \\
M=\beta M+(1-\beta) K\left[\left(\frac{h P}{2 K M}\right)^{2}-1\right] .
\end{gathered}
$$

By rearranging the two equations in terms of $P$ and $M$, we get

$$
\frac{3}{2} P^{2}-\frac{2 K}{h} M=0, \quad M^{3}+K M^{2}-\frac{h^{2}}{4 K} P^{2}=0 .
$$

Plugging the first equation into the second and rearranging the terms, we get

$$
M\left(M^{2}+K M-\frac{h}{3}\right)=0 .
$$

Thus, either $M=0$ or $M=\Phi / 2$ provides the solution to the equation above. However, the first solution does not constitute an interior point (refer to Proposition 5). On the other hand, the second solution is indeed positive $(\because \Phi>0)$ and constitutes the interior equilibrium above. $\bar{P}$ in (11) comes from the relationship $(3 / 2) P^{2}=(2 K / h) M$ above.

Proposition 4. Suppose that $9 \Phi^{4}-6 \Phi^{2}-64 h^{2}>0$ and $(16 / 15) h<K^{2}$. If $\alpha<(5 / 3)-\left((1+\beta) \Phi^{2} / 4 h(1-\beta)\right)$ and $\beta<\left(20 h-3 \Phi^{2}\right) /\left(20 h+3 \Phi^{2}\right)$, then the fixed point in Proposition 3 is unstable.

Proof. In this proof, we suppress the time index $t$ for enhanced readability. We first consider linear approximation of the dynamics around the interior equilibrium. Applying the Taylor approximation to $((10 a)$ and $(10 b))$, we get the following matrix that governs the dynamics around an arbitrary point in the state space:

$$
\left[\begin{array}{cc}
\frac{3 \alpha-1}{2}-\frac{2 K(1-\alpha)}{h} \cdot \frac{M}{P^{2}} & \frac{2 K(1-\alpha)}{h} \cdot \frac{1}{P} \\
\frac{h^{2}(1-\beta)}{2 K} \cdot \frac{P}{M^{2}} & \beta-\frac{h^{2}(1-\beta)}{2 K} \cdot \frac{P^{2}}{M^{3}}
\end{array}\right] .
$$

In particular, the matrix reduces to the following one around the interior fixed point $(\bar{P}, \bar{M})$ :

$$
\left[\begin{array}{cc}
1 & (1-\alpha) \sqrt{\frac{6 K}{h \Phi}} \\
(1-\beta) \sqrt{\frac{1}{3 K} \cdot\left(\frac{2 h}{\Phi}\right)^{3}} & \beta+\frac{4 h(1-\beta)}{3 K \Phi-2 h}
\end{array}\right] .
$$

Note that $(3 / 2) \Phi^{2}=2 h-3 K \Phi$. Then, the characteristic equation of the matrix above comes as follows:

$$
\begin{aligned}
\lambda^{2}+ & \left(\frac{8 h(1-\beta)}{3 \Phi^{2}}-1-\beta\right) \lambda+\beta \\
& +\frac{4 h(1-\beta)(3 \alpha-5)}{3 \Phi^{2}} \\
= & \lambda^{2}+\Theta \lambda+\Omega=0,
\end{aligned}
$$


where $\Theta \equiv\left(8 h(1-\beta) / 3 \Phi^{2}\right)-1-\beta$ and $\Omega \equiv \beta+(4 h(1-$ $\left.\beta)(3 \alpha-5) / 3 \Phi^{2}\right)$ denote two constants for simplification of the following expressions. The condition that the equation has two distinctive real roots $\lambda_{1}$ and $\lambda_{2}$ is $\Theta^{2}>4 \Omega$, which is summarized as below by a chain of arithmetic operations:

$$
\begin{aligned}
& 64 h^{2}(1-\beta)^{2}+9 \Phi^{4}(1+\beta)^{2} \\
&> 12 \Phi^{2}\{4 h(1-\beta)(1+\beta)+\beta\} \\
&+16 h(1-\beta)(3 \alpha-5) .
\end{aligned}
$$

Since the second term (the last term) in the right-hand side is negative, it suffices that $64 h^{2}(1-\beta)^{2}+9 \Phi^{4}(1+\beta)^{2}>$ $12 \Phi^{2}\{4 h(1-\beta)(1+\beta)+\beta\}$ holds. Applying another arithmetic operation and collecting terms reorganize this relation as follows:

$$
\begin{aligned}
& \left(3 \Phi^{2}+8 h\right)^{2} \beta^{2}-2\left(64 h^{2}-9 \Phi^{4}+6 \Phi^{2}\right) \beta+\left(3 \Phi^{2}-8 h\right)^{2} \\
& \quad>0 .
\end{aligned}
$$

Therefore, if $9 \Phi^{4}-6 \Phi^{2}-64 h^{2}>0$, then the inequality above holds and the characteristic equation has two distinctive real solutions.

Now, using the facts that $\lambda_{1}+\lambda_{2}=-\Theta$ and $\lambda_{1} \lambda_{2}=\Omega$, we can find sufficient conditions which establish stability or instability of the interior equilibrium. Indeed, if $\lambda_{1} \lambda_{2}<-1$, then at least one eigenvalue is larger than 1 or less than -1 , thereby making the system around the interior fixed point unstable. Since $\lambda_{1} \lambda_{2}=\Omega=\beta+4 h(1-\beta)(3 \alpha-5) / 3 \Phi^{2}$, the condition $\lambda_{1} \lambda_{2}<-1$ reduces into the following inequalities:

$$
\begin{aligned}
& 3 \beta \Phi^{2}+4 h(1-\beta)(3 \alpha-5)<-3 \Phi^{2} \\
& \text { or } \quad \alpha<\frac{5}{3}-\frac{(1+\beta) \Phi^{2}}{4 h(1-\beta)} .
\end{aligned}
$$

The right-hand side of the inequality for $\alpha$ is positive if $\beta<\left(20 h-3 \Phi^{2}\right) /\left(20 h+3 \Phi^{2}\right)$. The right-hand side of the inequality for $\beta$ is also positive under the condition of $(16 / 15) h<K^{2}$. Thus, two conditions pertaining to $\alpha$ and $\beta$ are well established as a sufficient condition for the interior equilibrium to be unstable, which completes the proof.

The proof needs to be complemented since Proposition 4 provides only a sufficient condition. We know that if $\mid \lambda_{1}+$ $\lambda_{2} \mid<1$ and $\lambda_{1} \lambda_{2}>0$ then all the eigenvalues are less than 1 , thereby making the system around the interior fixed point stable. However, one can show in a similar way that there is no $\lambda_{i}$ that satisfies those conditions. Indeed, since $\lambda_{1}+\lambda_{2}=$ $-\Theta=1+\beta-\left(8 h(1-\beta) / 3 \Phi^{2}\right)$, the condition $\left|\lambda_{1}+\lambda_{2}\right|<1$ reduces to $(6 K \Phi /(6 h-3 K \Phi))<\beta<(4 h /(6 h-3 K \Phi))$. On the other hand, the condition $\lambda_{1} \lambda_{2}=\Omega>0$ reduces to $\alpha>(5 / 3)-\left(\beta \Phi^{2} / 4 h(1-\beta)\right)$ and $\beta>(4 h /(6 h-3 K \Phi))$, which cannot be compatible with the previous condition. A similar type of incompatibility occurs in the case of $\lambda_{1} \lambda_{2}>$ 1. This possibility of instability is also frustrated since the

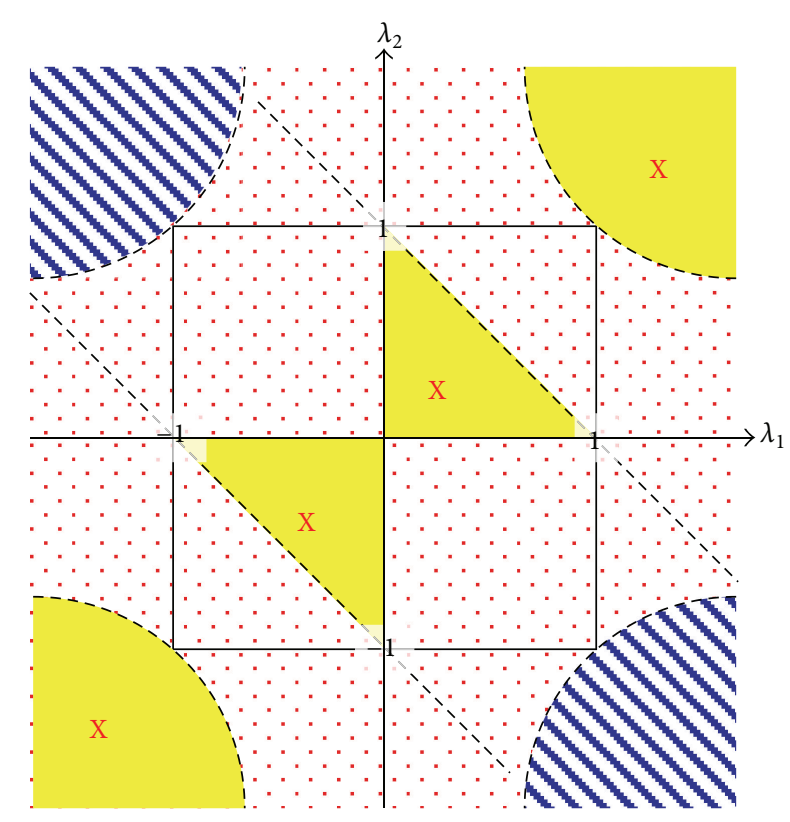

FIGURE 1: Regions of the $\lambda_{1}-\lambda_{2}$ space: explored and unexplored.

condition $\lambda_{1} \lambda_{2}>1$ requires either $\alpha>1$ or $\beta>1$, which is not acceptable. As a result, we have explored the following regions of the $\lambda_{1}-\lambda_{2}$ space in Figure 1 . The shaded regions with " $X$ " mark are the subsets whose $\left(\lambda_{1}, \lambda_{2}\right)$-tuples cannot be realized in our model. The two hatched regions depict the sufficient conditions in this proposition. Thus, Proposition 4 leaves some regions unexplored.

Proposition 3 reveals that there is a unique interior equilibrium if any one exists. Proposition 4 , however, implies that the interior equilibrium is highly likely unstable. Though there remain some regions that should be explored in the $\lambda_{1}-\lambda_{2}$ space (see Figure 1), it may not be a good strategy to investigate the stability regions first, with a vague hope to discover such a case. In fact, it seems hard to find an instance in which the interior equilibrium is stable (refer to some experimental results in Section 5). And if this is true, one needs to analyze another possibility and different aspects of the dynamics.

Since the $P(t)-M(t)$ state space is bounded, the system dynamics may have a boundary equilibrium that needs to be investigated. Obviously, for example, $(0,0)$ cannot be a boundary fixed point which represents a complete collapse of both market and industry (Also note that the dynamic equations ((10a) and $(10 \mathrm{~b}))$ are not defined at $(0,0))$. However, the system state may approach the origin very closely (refer to the proposition below and the discussions in the next section). Furthermore, neither $(z, 0)$ with any positive $z \in$ $(0, N]$ nor $(0, z)$ with $z \in\left(0, M_{\max }\right]$ can be a Nash equilibrium since, at least for a (potential) player, a deviation from the corresponding state improves its gain. For example, an active player at $(N, 0)$ will be better off if it leaves the market and avoids a negative payoff. The following proposition deals with the other types of possible boundary equilibria.

Proposition 5. The following two states are (near) boundary fixed point equilibria (compatible with the static model as in 
Proposition 1). However, both are unstable under the certain conditions (if any) specified below.

(i) For an arbitrary small $\varepsilon,(\varepsilon, \varepsilon)$ is a near boundary Nash equilibrium. However, $(\varepsilon, \varepsilon)$ is unstable if $h>2 \mathrm{~K}$.

(ii) $\left(N, M_{\max }\right)$ is a boundary Nash equilibrium if $q_{N} \leq$ $\left(2 K M_{\max } /(N+1)\right)+(N / 2)$. However, $\left(N, M_{\max }\right)$ is unstable if $4 K M_{\max }<3 h N^{2}$ and $h^{2} N^{2}<$ $4 K M_{\max }^{2}\left(K+M_{\max }\right)$.

Proof. It is easy to show that the two states asserted above become Nash equilibria in the sense compatible with Proposition 1 . For $\varepsilon$ is very close to 0 , for example, $(\varepsilon, \varepsilon) \approx$ $(0,0)$ implies that any potential provider (even the most efficient provider) outside of the industry cannot expect any positive gain from joining the industry. Thus, the best response of all the providers is to stay out of the industry. Similarly, in the other extreme cases of $\left(N, M_{\max }\right)$, where all the providers join the industry, even the least efficient provider enjoys a positive gain from performing as much marketing effort as determined by Proposition 1 if $q_{N} \leq$ $\left(2 K M_{\max } /(N+1)\right)+(N / 2)$. Therefore, no provider will leave the industry and $M_{\max }$ is sustained.

In order to show the instability of $(\varepsilon, \varepsilon)$, we first apply the Taylor expansion to $P(t+1)-P(t)$ and $M(t+1)-M(t)$ at $(\varepsilon, \varepsilon)$ and get

$$
\begin{aligned}
& P(t+1)-P(t) \\
& \quad=(1-\alpha)\left\{\left(\frac{2 K}{h \varepsilon}-\frac{3}{2}\right) P(t)+\frac{2 K}{h \varepsilon} M(t)+\frac{2 K}{h}\right\}-\frac{\alpha \varepsilon}{2}
\end{aligned}
$$

$$
\begin{aligned}
M( & +1)-M(t) \\
= & (1-\beta) \\
& \times\left\{\frac{h^{2}}{2 K \varepsilon} P(t)-\left(1+\frac{h^{2}}{2 K \varepsilon}\right) M(t)+\frac{h^{2}}{4 K}-K\right\}+\varepsilon .
\end{aligned}
$$

Now, we incorporate a small perturbation around $(\varepsilon, \varepsilon)$ into ((21a) and (21b)). We will show that, for at least one type of perturbation, both $P(t+1)-P(t)$ and $M(t+1)-M(t)$ become positive, which means that the system state grows apart from the near boundary equilibrium $(\varepsilon, \varepsilon)$. For example, we take into account a small perturbation in a north-east direction such as $\left(\varepsilon+\varepsilon_{1}, \varepsilon+\varepsilon_{2}\right)$ where both $\varepsilon_{1}$ and $\varepsilon_{2}$ are positive and sufficiently small. Then, after a series of arranging and collecting terms, ((21a) and (21b)) reduce as follows:

$$
\begin{aligned}
& P(t+1)-P(t) \\
& \quad=\frac{2 K(1-\alpha)}{h}\left(3+\frac{\varepsilon_{1}+\varepsilon_{2}}{\varepsilon}\right)+\left(\alpha-\frac{3}{2}\right) \varepsilon+\frac{3}{2}(\alpha-1) \varepsilon_{1}
\end{aligned}
$$

$$
\begin{aligned}
& M(t+1)-M(t) \\
& \quad=(1-\beta)\left\{\frac{h^{2}}{2 K}\left(\frac{\varepsilon_{1}-\varepsilon_{2}}{\varepsilon}+\frac{1}{2}\right)-K\right\}+\beta\left(\varepsilon+\varepsilon_{2}\right)-\varepsilon_{2} .
\end{aligned}
$$

The first term in the right-hand side of (22a) is always positive and the other two terms are arbitrary small enough for the overall magnitude of $P(t+1)-P(t)$ to be positive. If $\varepsilon_{1}>\varepsilon_{2}$ and the condition in (i) holds, then the first two terms in the right-hand side of (22b) are positive. Thus, there are many directions in the north-east area of $(\varepsilon, \varepsilon)$ along which both $P(t+1)-P(t)$ and $M(t+1)-M(t)$ become positive (One can also prove that both (21a) and (21b) lead to a divergence from $(\varepsilon, \varepsilon)$ in the other areas, e.g., a south-east direction of $(\varepsilon, \varepsilon))$.

One can apply a similar procedure to the case of the boundary equilibrium $\left(N, M_{\max }\right)$. First, the dynamics around $\left(N, M_{\max }\right)$ can be written as follows:

$$
\begin{aligned}
& P(t+1)-P(t) \\
& =(\alpha-1)\left\{\left(\frac{3}{2}+\frac{2 K M_{\max }}{h N^{2}}\right) P(t)\right. \\
& \left.\quad-\frac{2 K}{h N} M(t)-\frac{2 K M_{\max }}{h N}\right\} \\
& M(t+1)-M(t) \\
& =(1-\beta)\left\{\frac{h^{2} N}{2 K M_{\max }^{2}} P(t)-\left(1+\frac{h^{2} N^{2}}{2 K M_{\max }^{3}}\right) M(t)\right. \\
& \left.+\frac{h^{2} N^{2}}{4 K M_{\max }^{2}}-K\right\} .
\end{aligned}
$$

To determine its stability, we again evaluate a small perturbation around $\left(N, M_{\max }\right)$. In this case, however, one needs to examine only one direction of perturbation, that is, $(N-$ $\left.\varepsilon, M_{\max }-\varepsilon\right)$, where $\varepsilon$ is positive and sufficiently small. After a series of operations, ((23a) and (23b)) reduce as follows:

$$
\begin{aligned}
& P(t+1)-P(t) \\
& =(1-\alpha)\left\{\frac{4 K M_{\max }-3 h N^{2}}{2 h N}\right. \\
& \left.+\left(\frac{2 K\left(M_{\text {max }}-N\right)}{h N^{2}}+\frac{3}{2}\right) \varepsilon\right\} \\
& M(t+1)-M(t) \\
& =(1-\beta)\left\{\frac{h^{2} N^{2}-M_{\max }-K}{4 K M_{\max }^{2}}\right. \\
& \left.+\left(\frac{h^{2} N\left(N-M_{\max }\right)}{2 K M_{\max }^{3}}+1\right) \varepsilon\right\} .
\end{aligned}
$$

Note that the last terms in the respective right-hand side of $((24 \mathrm{a})$ and $(24 \mathrm{~b}))$ are to be arbitrary small. Thus, the signs of 
$P(t+1)-P(t)$ and $M(t+1)-M(t)$ are determined by their first terms, each of which is negative if the conditions in (ii) hold. As a result, both $P(t+1)-P(t)$ and $M(t+1)-M(t)$ become negative, which establishes the instability of $\left(N, M_{\max }\right)$.

First note that the near boundary equilibrium $(\varepsilon, \varepsilon)$ represents a virtual collapse of the market as well as the industry. On the other hand, the boundary equilibrium $\left(N, M_{\max }\right)$ represents a perfect prosperity of the business ecosystem. Thus, the stability analysis about those two boundary states suggests not only good news but also bad news. The good news is that as both the market force and the number of providers approach nil, at least a tiny fraction of providers still seize the opportunity to earn positive gains, which would in turn boost the market demand. This mechanism of escaping from almost complete destruction comes from the structural property of the dynamics that would not allow nil to be as a fixed point.

On the other hand, the instability of the complete prosperity presents a bad news. As shown in the proof, a small perturbation occurring around the boundary state may lead the system away from the ideal situation. This system behavior probably comes from the mechanism around the saturation state $\left(N, M_{\max }\right)$. That is, with a shock making the system deviate from the saturation, some marginal providers on the limit of positive gain at the saturation now would experience a loss and leave the industry. This reduction of the number of providers is highly likely to cut back the market value, which would in turn disturb more providers on the verge of leaving the industry.

These results suggest that $P(t)$ and $M(t)$ which are sufficiently small [or close to their respect upper bounds] may approach the origin [or the saturation] over time but suddenly explodes near the origin [or shrink back near the saturation]. Thus, the stability analysis so far hints a possible cyclic solution to our dynamics. We will check out this possibility through numerical experiments in the next section.

Even though it is true that our system behavior has the property of natural resilience from deterioration, it may stay at the states near a collapse over a long period of time. Thus, we need to develop a policy measure that protects the system from moving toward the collapse and establishes a locally stable positive equilibrium that attracts system trajectories generated around it. If it is successful, the market and the industry will not only be sustained but also stabilized over time at some desirable states. We will also deal with this issue in the next section.

\section{Experiments and Discussions}

We conduct some numerical simulations in order to examine more thoroughly the system behaviors in various scenarios. In our simulations, both $P(t)$ and $M(t)$ are endogenously determined according to the system dynamics presented in the previous section. Subsequently, the total marketing efforts are also changing as the system states vary, which provides a sharp contrast with the theoretical results in the static model

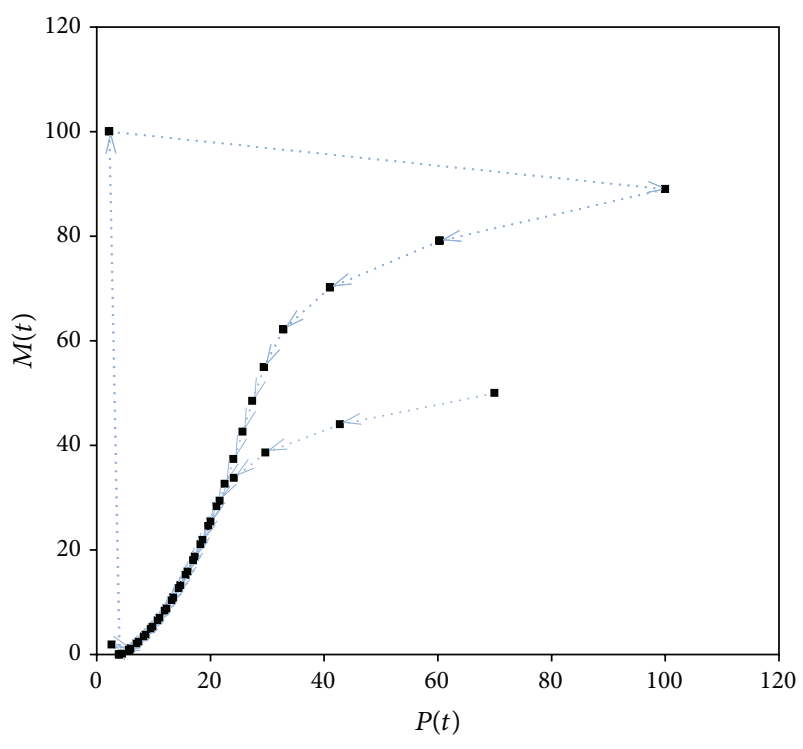

Figure 2: Experimental example. Here, $\alpha=0.7, \beta=0.9, K=10$, and $h=1$. The initial point is $P_{0}=70$ and $M_{0}=50$ with $N=100$ and $M=100$. Arrows show the directions of the migration of the system state. The system state moves toward the origin after leaving the initial point. Interestingly, however, there is a loop starting from near the origin. The presence of the loops implies a cycle in the system behavior.

such as Kim's study [27] (This finding (and the followings) can be captured only in a dynamic setting).

As Propositions 3 to 5 reveal, the outcomes of the dynamic model are quite different from the ones of the static model described in Proposition 1. First of all, note that $(\varepsilon, \varepsilon)$, close to a complete collapse of the market and the industry, is not a stable equilibrium, but the system is highly likely to approach the origin. Thus, ToC (the tragedy of the commons) seems to be inevitable in some situations, particularly when the market is not mature (i.e., small $M_{0}$ ) with many providers (i.e., large $\left.P_{0}\right)$. Figure 2 depicts this possibility.

As raised in the previous section, a cycle or a sequence of periodic points seems to constitute a solution to our dynamic equations. In fact, the emergence of the ToC trap and the oscillation dynamics are neither a new phenomenon nor an unusual feature in dynamic models, particularly on the basis of structured interactions among agents (e.g., Perc and Szolnoki [21], Perc et al. [22], and Santos et al. [23]) (In particular, the emergence of cycles (or oscillations) has been observed in the context of adaptive networks (Szolnoki and Perc [24, 25]) and with three competing strategies (Szolnoki et al. [28]), as well as under a presence of punishment (Szolnoki et al. [29]) or a reward (Szolnoki and Perc [26]). However, our model does not assume a structured population; it is one of our future research topics to incorporate organized interactions among providers (see also our comment in the future works)). It is also well known that finding such a cycle is usually a very difficult task, and our model is no exception. However, our numerical experiments including the one above confirm (or at least indicate) that the cyclic solution commonly occurs for many initial states. For example, the experiment outcome 


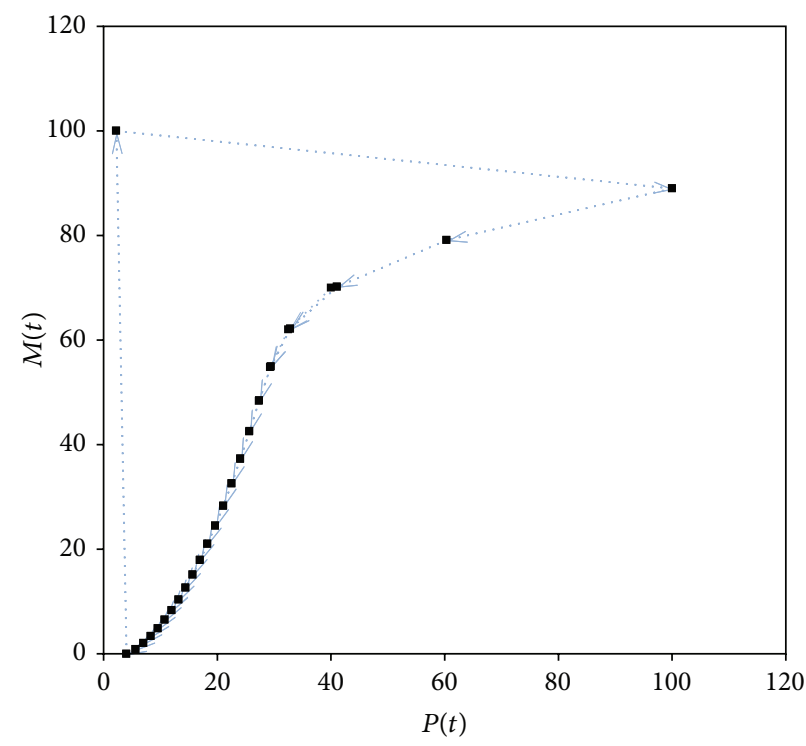

Figure 3: A cycle example. Here, all the parameters remain the same as the ones in Figure 2 except the initial states. As starting points, $P_{0}=40$ and $M_{0}=70$ are applied to this example.

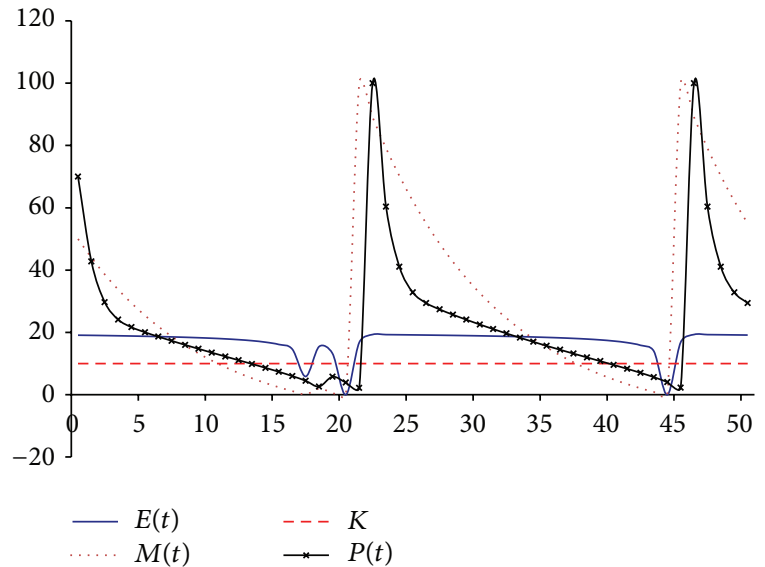

(a) $P_{0}=70$ and $M_{0}=50$

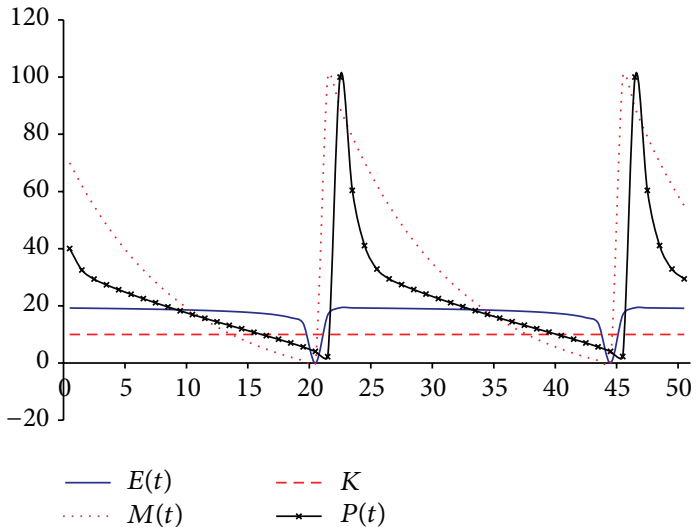

(b) $P_{0}=40$ and $M_{0}=70$

Figure 4: Behaviors of key attributes of system. Here, all the parameters and the initial states remain the same as the ones in Figure 2 for (a) and Figure 3 for (b). The time series graphs of $P(t)$ and $M(t)$ also clearly point out the cyclic pattern of the system behavior even though they look a little different in the first period due to different starting points. In both instances, the total marketing efforts $E(t)$ are higher than their optimal level $K$ most of the time.

depicted in Figure 3 shows a clear cycle of the particular dynamics specified in Figure 2 (except the initial point). In this instance, the initial state is deliberately set to $\left(P_{0}, M_{0}\right)=$ $(40,70)$, which closely lies on the cycle observed in Figure 2.

Moreover, Figure 4 represents the system behavior along the timeline. They show clear cycles repeating over time. In most of time, the total marketing efforts remain higher than the optimal level $K$. Thus, the market maturity eventually drops down to almost zero, which in turn results in deterioration of providers' gain, that is, the ToC trap. Whenever the market maturity approaches nil, most efficient few providers survive and are able to sweep all the gains from the market. Thanks to the monopolized benefits, they can boost the market and the maturity rapidly increases. This explosion of the market opportunity attracts more providers, and the new cycle starts thereafter.

If this is the most common case then the remaining issue should be related to the ToC trap which seems inevitable in the cycle. In particular, we need to develop a remedy (a policy measure) to cope with ToC. One possible way to pursue is to introduce a regulation that makes providers keep the marketing efforts at their reasonable levels (probably coupled with their cost efficiencies). We may also implement a policy that puts some barriers to preserve a minimum level of the market maturity. Figure 5 shows the effect of the latter approach. One may consider a similar barrier set up for $P(t)$ 


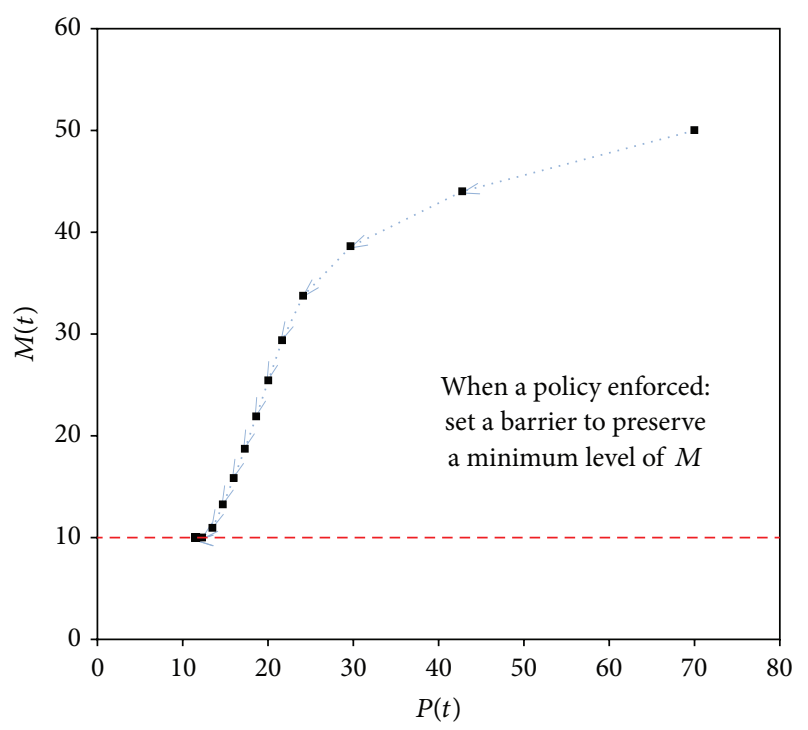

FIgURE 5: Experimental example, with a barrier to sustain the market. Here, all the parameters and the initial states remain the same as the ones in Figure 2 (but the axes have been scaled differently). In addition, however, there is a barrier set up for the market maturity. This barrier keeps the market maturity factor $M(t)$ above 10 . Arrows show the directions of the migration of the system state, which settles down to a fixed state, of which $M(t)$ corresponds to the barrier. In this instance, there is no cycle.

and compare the outcomes of two approaches in terms of social welfare (As a matter of fact, we also tried this approach but omitted the results here since the outcomes of this policy do not seem to follow a rule; they produce nonstructured many small cycles. Thus, apparently (not rigorously), the policy to sustain at least a certain number of providers fails in providing better outcomes).

\section{Conclusion and Future Works}

This study examined the possibilities of a disruption of a business ecosystem, so-called the ToC trap, which describes the situation where the total marketing efforts $\bar{E}$ exceed their socially optimal level $K$. Not only the interior but also the boundary equilibria were found and analyzed for their stability. We also conducted some numerical experiments and discovered cycles as solutions to some particular instances. Since those cycles contain the ToC traps, we simulated a policy measure to maintain a minimum level of market maturity and checked out the effectiveness of the policy. Even though we had in mind some ICT industries (e.g., the social commerce industry) with fierce marketing competition, our approach and results will not be confined to the ICT industries. And we hope this study helps to find a clue to avoid the $\mathrm{ToC}$ trap, thereby shedding new light on the sustainable growth of business ecosystems.

However, the analytical results and implications have been established on the basis of our modeling assumptions, and they may not be generalized into all the situations. In particular, our assumption of perfectly mixed (or randomized) interactions among the players is not a fully realistic one. This remains as one of the limitations of our model and approach. Thus, it will be an obviously interesting direction of our next research step to extend the proposed game model with a structured population of providers (for benchmarking studies, refer to Szolnoki et al. [28], Szolnoki et al. [29], and Wang et al. [30]).

In our future works, we will also refine policy measures that were derived in the course of implementing our suggestions to alleviate the risk of the ToC trap. We will incorporate various regulatory schemes and other policy ideas (e.g., Greely [31]) and evaluate their effects on the longrun equilibrium. Lastly, we will compare the policy outcomes with one generated from a hypothetical social planner who determines the total marketing level that optimizes the entire market value. Case studies together with statistical analysis will be conducted in order to test these possibilities in practice and to investigate the effects of the parameters on expected results.

\section{Conflict of Interests}

The author declares that there is no conflict of interests regarding the publication of this paper.

\section{References}

[1] D. MacMillan, "Groupon's stumbles may force it to pare back size of IPO," Bloomberg, 2011, http://www.bloomberg.com.

[2] D. MacMillan, "LivingSocial aims to be different from Groupon," Business Week, 2011, http://www.businessweek.com.

[3] D. MacMillan, "Groupon China venture said to fire workers for poor performance," Bloomberg, 2011, http://www.bloomberg.com.

[4] D. Reibstein, "How sustainable is Groupon's business model?" Knowledge at Wharton, 2011, http://knowledge.wharton.upenn .edu.

[5] K. Webster, "Groupon's business model: bubble or the real deal?" 2011, http://pymnts.com.

[6] R. Wheeler, "Groupon gone wrong! Harvard business fellow's warning to investors and entrepreneurs," 2011, http:// pymnts.com.

[7] V. Kumar and B. Rajan, "Social coupons as a marketing strategy: a multifaceted perspective," Journal of the Academy of Marketing Science, vol. 40, no. 1, pp. 120-136, 2012.

[8] K. Patel, “Groupon marketing spending works almost too well," Ad Age Digital, 2011, http://adage.com.

[9] M. A. Cusumano, "Technology strategy and management platform wars come to social media," Communications of the ACM, vol. 54, no. 4, pp. 31-33, 2011.

[10] N. Kruschwitz, "Size matters in social business adoption," MIT Sloan Management Review, 2012, http://sloanreview.mit.edu.

[11] J. Leber, “The biggest cost of Facebook's Growth," Technology Review, 2012, http://www.technologyreview.com.

[12] M. Anderson, J. Sims, J. Price, and J. Brusa, "Turning "like" to "buy": social media emerges as a commerce channel," White Paper, Booz \& Company, 2012.

[13] B. Urstadt, "Social networking is not a business," MIT Technology Review, 2008, http://www.technologyreview.com. 
[14] M. Lodefalk, "Servicification of manufacturing: evidence from Sweden," International Journal of Economics and Business Research, vol. 6, no. 1, pp. 87-113, 2013.

[15] R. Schmitt and S. Hatfield, "Strategic servicification-a quality based approach beyond service-engineering," in Manufacturing Systems and Technologies for the New Frontier, M. Mitsuishi, K. Ueda, and F. Kimura, Eds., pp. 511-514, 2008.

[16] A. T. Stephen and O. Toubia, "Deriving value from social commerce networks," Journal of Marketing Research, vol. 47, no. 2, pp. 215-228, 2010.

[17] J. Alroy, "A multispecies overkill simulation of the endPleistocene megafaunal mass extinction," Science, vol. 292, no. 5523, pp. 1893-1896, 2001.

[18] G. Hardin, “The tragedy of the commons," Science, vol. 162, no. 3859, pp. 1243-1248, 1968.

[19] G. M. Greco and L. Floridi, "The tragedy of the digital commons," Ethics and Information Technology, vol. 6, no. 2, pp. 7381, 2004.

[20] Knowledge at Wharton, "Dot-com bubble, part II? Why it's so hard to value social networking sites?" Knowledge at Wharton, 2006, http://knowledge.wharton.upenn.edu.

[21] M. Perc and A. Szolnoki, "Coevolutionary games-a mini review, BioSystems, vol. 99, no. 2, pp. 109-125, 2010.

[22] M. Perc, J. Gómez-Gardeñes, A. Szolnoki, L. M. Floría, and Y. Moreno, "Evolutionary dynamics of group interactions on structured populations: a review," Journal of the Royal Society Interface, vol. 10, no. 80, Article ID 20120997, 2013.

[23] F. C. Santos, J. M. Pacheco, and T. Lenaerts, "Evolutionary dynamics of social dilemmas in structured heterogeneous populations," Proceedings of the National Academy of Sciences of the United States of America, vol. 103, no. 9, pp. 3490-3494, 2006.

[24] A. Szolnoki and M. Perc, "Resolving social dilemmas on evolving random networks," Europhysics Letters, vol. 86, no. 3, Article ID 30007, 2009.

[25] A. Szolnoki and M. Perc, "Emergence of multilevel selection in the prisoner's dilemma game on coevolving random networks," New Journal of Physics, vol. 11, no. 9, Article ID 093033, 2009.

[26] A. Szolnoki and M. Perc, "Evolutionary advantages of adaptive rewarding," New Journal of Physics, vol. 14, no. 9, Article ID 093016, 2012.

[27] D. Kim, "A game theoretic analysis of social commerce ecosystem at the crossroads," Asia Pacific Journal of Information Systems, vol. 23, no. 2, pp. 67-86, 2013.

[28] A. Szolnoki, M. Perc, and G. Szabó, "Phase diagrams for threestrategy evolutionary prisoner's dilemma games on regular graphs," Physical Review E. Statistical, Nonlinear, and Soft Matter Physics, vol. 80, no. 5, Article ID 056104, 2009.

[29] A. Szolnoki, G. Szabó, and M. Perc, "Phase diagrams for the spatial public goods game with pool punishment," Physical Review E. Statistical, Nonlinear, and Soft Matter Physics, vol. 83, no. 3, Article ID 036101, 2011.

[30] Z. Wang, A. Szolnoki, and M. Perc, "Interdependent network reciprocity in evolutionary games," Scientific Reports, vol. 3, 2013.

[31] B. Greeley, "Should Google and the ad industry regulate themselves?” Business Week, 2012, http://www.businessweek.com. 


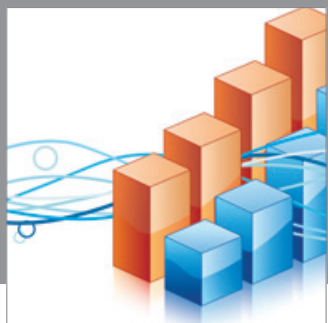

Advances in

Operations Research

mansans

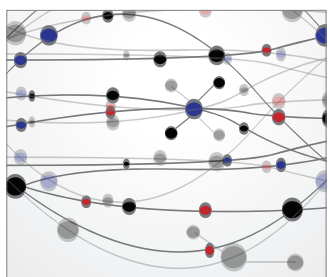

The Scientific World Journal
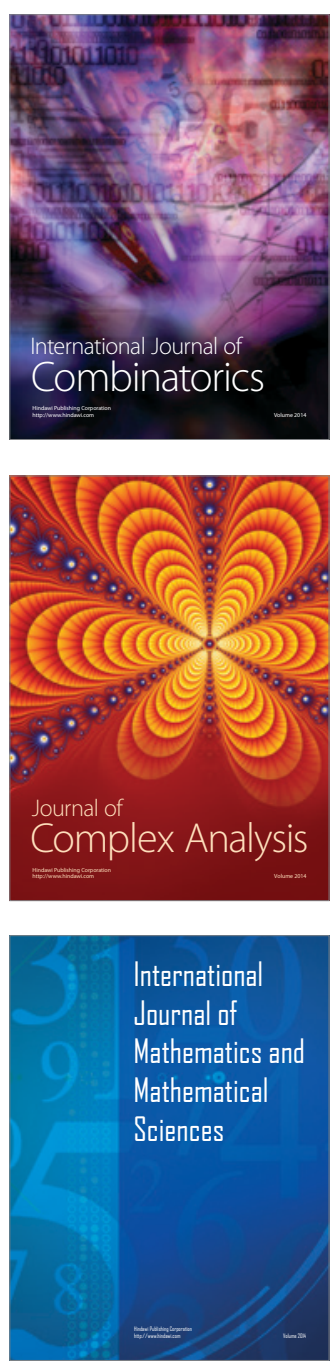
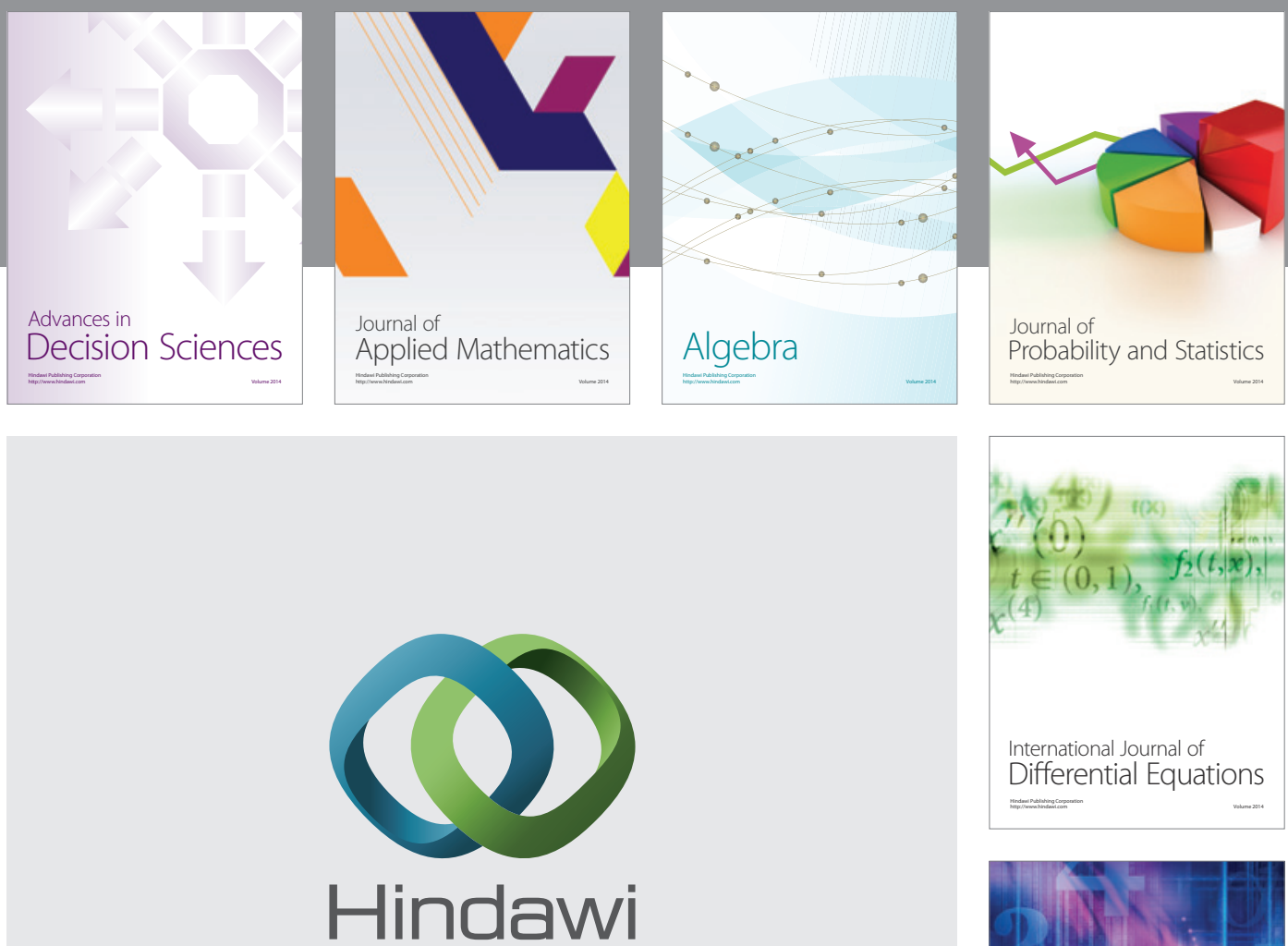

Submit your manuscripts at http://www.hindawi.com
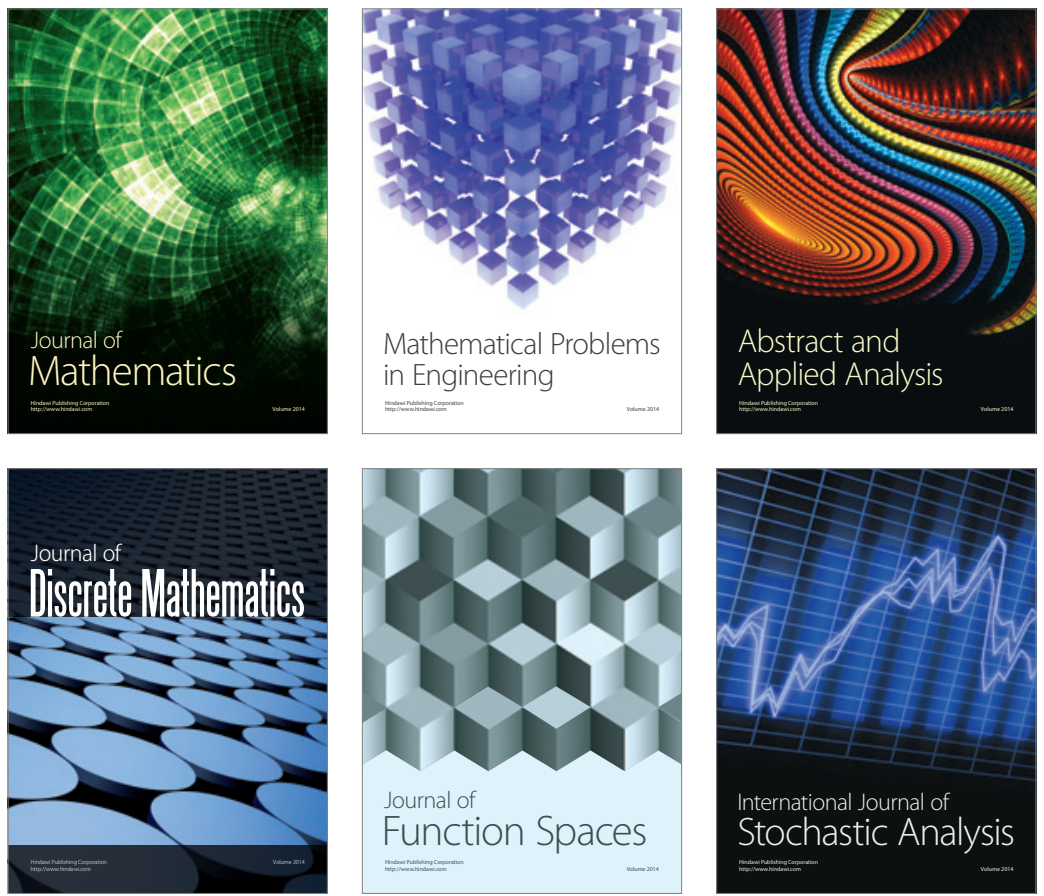

Journal of

Function Spaces

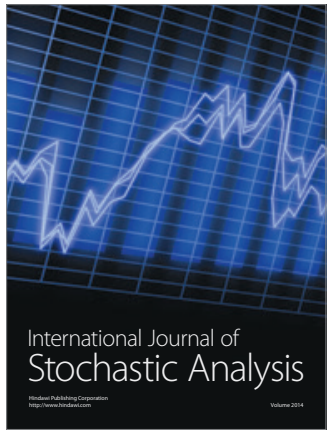

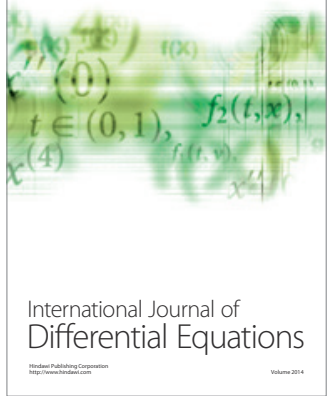
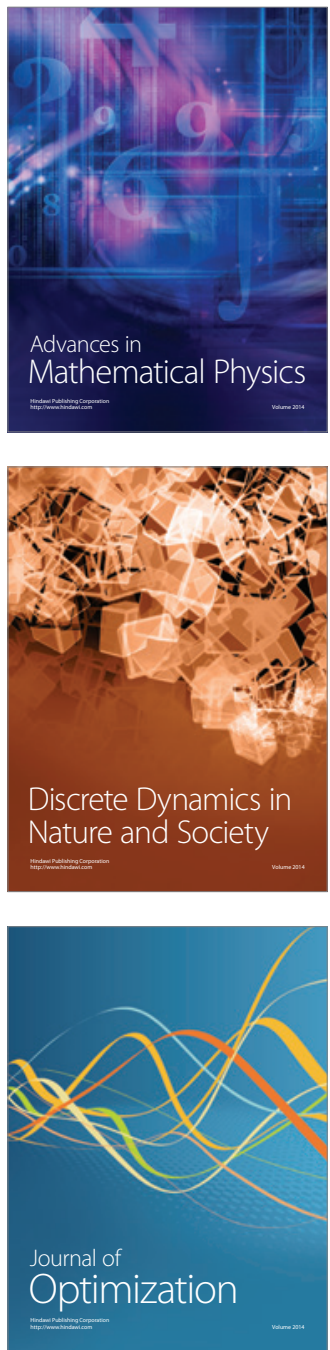